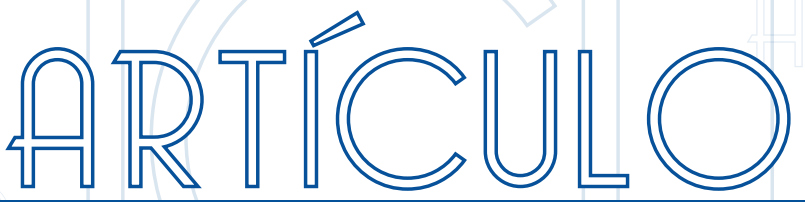

\section{INCIDENCIA DE LAS RUTAS DE CICLISMO EN LA DEMANDA DE TURISMO ACTIVO DE NATURALEZA DE LOS CICLISTAS QUE RECORREN LA PROVINCIA DEL GUAYAS. DISEÑO DE UN APLICATIVO MÓVIL DE CICLOTURISMO}

\author{
INCIDENCE OF THE CYCLING ROUTES IN THE DEMAND OF ACTIVE NATURE -BASED \\ TOURISM OF THE CYCLISTS THAT RIDE IN GUAYAS PROVINCE. DESIGN OF A CYCLE \\ TOURISM MOBILE APPLICATION
}

\begin{abstract}
Aceptación: 01/10/2017
Publicación: 25/11/2017
\end{abstract}

\section{Resumen}

El cicloturismo es un tema que se encuentra en auge por la cantidad de personas e instituciones que están promoviendo el uso de la bicicleta como medio de transporte sustentable. En el presente trabajo se analizó la incidencia de las rutas de ciclismo en la demanda de turismo activo de naturaleza de los ciclistas de la zona, realizando una investigación de campo en una muestra de las ciclo rutas, para diseñar un aplicativo móvil de cicloturismo. Adicionalmente, se realizaron encuestas a una muestra de 384 ciclistas de la provincia del Guayas y una revisión documental para encontrar las mejores prácticas de otros aplicativos móviles de cicloturismo que se utilizan en otros países. Entre los principales hallazgos se encontró que la comunicación inmediata y masificada entre los ciclistas es el mayor determinante para definir la decisión de considerar las rutas de ciclismo, y que consecuentemente podría incrementar la exposición de estas rutas a otros usuarios o turistas que utilizan la bicicleta para involucrarse en nuevos lugares rodeados de naturaleza.

Palabras clave: cicloturismo, ciclismo, ciclo rutas, turismo activo de naturaleza, bicicleta, aplicativo Móvil.

\section{Abstract}

Cycle tourism is an activity gaining more adepts due to the support of government institutions and people who are promoting the use of bicycle as a sustainable mean of transport. In the present work was to analyze the incidence of cycling routes in the demand for active nature tourism of the cyclists of this zone, carrying out a field investigation in a sample of the cycle routes, to design a mobile application of cycling tourism. In addition, surveys were conducted on a sample of 384 cyclists from the province of Guayas and a documentary review to find the best practices of other cycling mobile applications that are used in other countries. Among the main findings, it was found that the immediate and mass communication among cyclists is the major determinant in the decision to consider cycling routes, and consequently could increase the exposure of these routes to other users or tourists who use the bicycle to get involved in new places surrounded by nature.

Keywords: cycle tourism, cycling, cycle roads, active nature tourism, bicycle, mobile application.

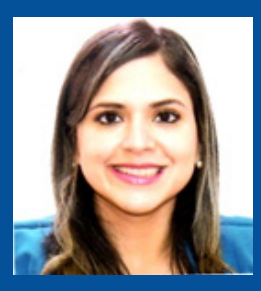

Ing. María Soledad Rea Fajardo, Mgs.

Doctorando en Ciencias de la Educación de la Universidad de La Habana (Cuba).

Magíster en Gestión de Organizaciones de la Université du Québec à Chicoutimi (Canadá) y la Universidad EAN (Colombia).

Diplomado Internacional en Evaluación de la Calidad de Programas de Educación Superior a Distancia, Universidad Técnica Particular de Loja. Ingeniera Comercial de la Universidad Católica de Santiago de Guayaquil.

Docente Titular de la Universidad Católica de Santiago de Guayaquil

E-mail: maria.rea@cu.ucsg.edu.ec soledadreaf@gmail.com

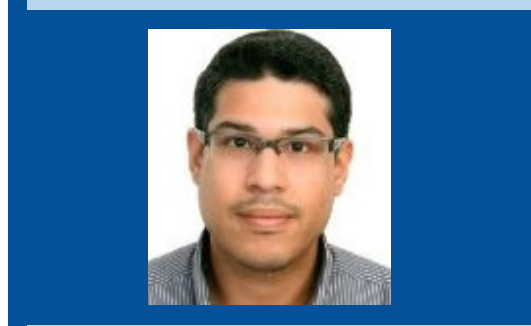

Econ. Luis Fernando Alban, Ms.C. Cursando el Doctorado en Ciencias de la Educación

Universidad de La Habana, Cuba Master of Science in Hotel, Restaurant and Tourism Management

Lester E. Kabacoff School of Hotel, Restaurant, and Tourism Administration, UNO

Bachelor in Science in Marketing and Business Administration, UNO

Docente, Investigador y Coordinador de Practicas Pre Profesionales,

Carrera de Ingeniería en Administración de Empresas Turísticas y Hoteleras

Facultad de Especialidades Empresariales

Consultor de Negocios de Alimentos y Bebidas Desarrollo de Negocios, Munizioni S.A

E-mail: luis.alban01@cu.ucsg.edu.ec

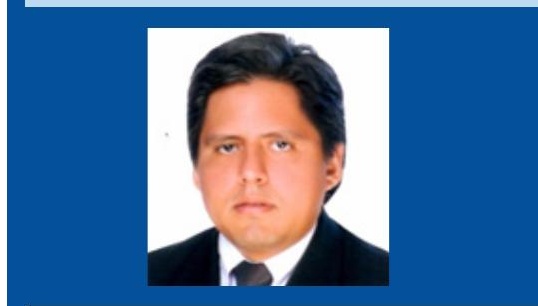

Econ. Pedro Cedeño Guerrero, Mgs.

Máster en Gestión de Proyectos de la Escuela Politécnica de Administración de Empresas ESPAE.

Analista Financiero, Especialista del Mercado Bursátil de la Corporación Financiera Nacional, BP.

E-mail: penacede@espol.edu.ec 


\section{Introducción}

El turismo activo de naturaleza tiene un impacto significativo en varios aspectos importantes en una localidad. Foszto (2014) indicó que el cicloturismo ayuda al desarrollo del turismo interno y externo, provee de un modelo ecológico sustentable y una revitalización económica y social a la región. El ciclismo se relaciona con ejes muy importantes, tales como el transporte, educación, innovación tecnológica, salud, deporte, inclusión social, turismo, respeto a la naturaleza, y otros dominios que se encuentran directamente relacionados con el Buen Vivir (Rea, Albán, Mori, y Cedeño, 2015). Por esto, el Ministerio de Transporte y Obras Públicas (2012) propuso el Plan Nacional de Ciclovías.

Se identificó como problemática principal el bajo nivel de demanda activa de turismo de naturaleza de los ciclistas que recorren la provincia del Guayas en el año 2015. Las Instituciones Públicas que fomentan el turismo se alejan cada vez mas de satisfacer aquellos turistas que buscan actividades de turismo activo en la provincia debido a que las ciclo rutas son poco conocidas.

El presente trabajo analizó la incidencia de las rutas de ciclismo en la demanda de turismo activo de naturaleza de los ciclistas que recorren la provincia del Guayas, realizando una investigación de campo en una muestra de las ciclo rutas, para diseñar un aplicativo móvil de cicloturismo. Adicionalmente, se realizaron encuestas a una muestra de los ciclistas que recorren la provincia del Guayas y una revisión documental para encontrar las mejores prácticas de otros aplicativos móviles de cicloturismo que se utilizan en otros países.

Según Vila, Brea y Carril (2012) el turismo activo está en crecimiento, es demandado por un tipo de turista practicante de tendencias actuales, exigente, informado e inmerso en una economía de experiencias que se trasladan al sector turístico, que desea nuevos productos turísticos o formas diferentes de practicar turismo. Es por ello que este turista necesita que se promueva la calidad de experiencia turística

Lo importante del desarrollo de un aplicativo de cicloturismo, será para que los ciclistas de la modalidad de aventura puedan contar con una guía virtual en tiempo real de la oferta vial para ciclistas en la Provincia del Guayas, ayudando así a personas que buscan usar la bicicleta con fines turísticos, a distinguir los riesgos, equipos, estado físico, y estar preparados para posibles encuentros con la flora y fauna de las zonas a visitar con la bicicleta.

Indirectamente la provincia contará con un método moderno de comunicación con sus turistas y la posible construcción de nuevas rutas temáticas y destinos específicos. Se prevé que los resultados promuevan las buenas prácticas de turismo consciente en la provincia como lo menciona Foszto (2014) debido a que el cicloturismo ayuda al desarrollo del turismo interno y externo, provee de un modelo ecológico sustentable y una revitalización económica y social a la región.

\section{Marco Teórico}

La bicicleta es prácticamente la misma desde fines del siglo 18, mientras alrededor de ella cambiaron modas, costumbres, tecnologías, ideologías, sistemas políticos y económicos. Hablar de este medio de transporte implica ante todo movilidad sostenible. Esta incide de varias formas desde la movilidad de los habitantes en sus desplazamientos diarios habituales hasta actividades recreativas y deportivas de visitantes y turistas.

De ahí la importancia de diversos planes de transporte ciclístico en diferentes ciudades del mundo. Ejemplos de ello se puede ver en Europa como son el Plan Andaluz y Eurovelo para el desarrollo del cicloturismo. Por lo tanto, las ciclo rutas son instrumentos planificadores básicos para el desarrollo de infraestructuras ciclistas. Algunos estudios investigativos realizados por la Consejería de Fomento y Vivienda de la Junta de Andalucía (2014) (en adelante, informe IECE), han determinado una relación directamente proporcional que cuanto más se usa la bicicleta como medio de transporte, mayor es el número de personas que practican cicloturismo. Vila et al. (2012) se enfocaron en el modelo de Antar-Ecotono, el mismo que define el campo del "Turismo de Naturaleza" y su subcampo "Turismo Activo"; a su vez este último posee tres campos de acción en el cual el CICLOTURISMO se ubica dentro de las actividades terrestres del mismo. En los últimos años se ha observado un incremento gradual del cicloturismo en numerosos países (Meschik, 2012), y un crecimiento notable en Norteamérica durante las dos últimas décadas (Pucher, Buehler, y Seinen, 2011). Este crecimiento se ha mantenido incluso en periodos de intensa crisis económica como la experimentada recientemente (Zovko, 2013). Se espera además un incremento continuado del mercado cicloturista (Mintel, 2009; Weston et al., 2012). Esta previsión es relevante debido al efecto económico multiplicador del cicloturismo, estimado en torno a 1,57 en Escocia (Zovko, 2013).

A este efecto habría que sumar el positivo impacto sobre la salud de las personas, con el consiguiente ahorro de gastos sanitarios. La relación coste-beneficio de la actividad física en bicicleta y pedestre es de 2,94, lo que significa que de cada dólar invertido se obtiene un beneficio sanitario de 2,94 dólares (Wang et al., 2005). (Fernández-Latorre, 2015).

Según el estudio encargado por el Parlamento Europeo sobre la Eurovelo (Weston et al., 2012) y descrito recientemente en el informe "El impacto económico del cicloturismo en Europa" (Consejería de Fomento y Vivienda de la Junta de Andalucía, 2014) se entiende por cicloturismo, aquellas visitas recreativas, ya sea durante más de un día o de solo un 
día fuera de casa, que implican el ciclismo de ocio como una parte fundamental o importante de la visita.

Se puede diferenciar tres tipos de cicloturismo: vacaciones ciclistas, centradas en el uso de la bicicleta (cycling holidays), ciclismo en vacaciones, donde se desarrolla esta actividad en las vacaciones (holiday cycling), y excursiones ciclistas de un día, consistentes habitualmente en paseos de un día o medio día (cycling day visit). En cualquiera de los casos, los ciclistas son turistas locales o extranjeros que experimentan la naturaleza, las atracciones culturales, los lugares especiales etnológicos y las regiones turísticas (Foszto, 2014).

Para que el cicloturismo ocurra se necesita de ciclo rutas, que según Gilabert (2015), se trata de una veintena de rutas de ciclismo que permiten combinar el uso de la bicicleta con la visita de lugares emblemáticos de la provincia o un territorio en específico y con fines específicos como ciclo rutas culturales (cicloturismo de paisajes naturales e historia), ciclo rutas recomendadas (descubiertas por ciclistas quienes descubren rutas accesibles y de escaso tráfico motorizado; en este sentido, se van a incluir algunas ciclo rutas habitualmente transitadas por ciclistas y que pueden tener una importancia de cara a mejorar la conectividad de otras rutas), las vías pecuarias (infraestructura para el cicloturismo y el ganando) y el senderismo como complemento del cicloturismo (toma como base el respeto al medio ambiente y la movilidad sostenible).

Vujko, Plavša y Ostojić (2014) demostraron el impacto que la ciclo ruta del Danubio podría tener en el desarrollo del cicloturismo usando el análisis de LoNGPESTEL. Ellos recolectaron la información directamente y aplicaron el método de encuestas a una muestra de 264 ciclistas de ambos géneros y 9 países diferentes, concluyendo que la montaña Fruška Gora tiene un gran potencial para el desarrollo del cicloturismo y que la ciclo ruta del Danubio puede ser su eje principal. La montaña Fruška Gora tiene como ventajas principales: su movilidad terrestre, viabilidad y fácil acceso desde muchos puntos de la montaña, así como debido a condiciones favorables, muchos valores naturales y culturales y numerosas actividades de entrenamiento, complementan este lugar.

Algunos autores como Piket, Eijgelaar y Peeters (2013) investigaron sobre el aspecto financiero del cicloturismo y presentaron un modelo de impacto económico basado en los gastos directos generados en las ciclo rutas europeas. Ellos encontraron que los cicloturistas gastan diariamente de forma similar a otros tipos de turistas, y que el cicloturismo puede contribuir significativamente en particular a economías rurales que no se han adherido previamente a la corriente principal del desarrollo por el turismo. Además, que tiene un impacto negativo al ambiente muy por debajo, en relación a otros tipos de turismos.
Tabla 1.Turismo de Naturaleza

\section{Turismo de Naturaleza}

\section{Ecoturismo}

Visitas guiadas

Observación / interpretación de la naturaleza - Actividades de educación ambiental

Observación de aves

Observación de cetáceos

Fotografía de naturaleza Ecoturismo científico

\section{Turismo Activo}

\begin{tabular}{|l|l|l|}
\hline Actividades & Área de marcha - & Esquí de travesía - \\
& Alpinismo/escala & Esquí de fondo \\
& Marcha a caballo & Raquetas \\
& - Ciclomontañismo & Perros con trineo \\
& Espeleología & - Motos de nieve \\
& Cicloturismo & Tiro con arco \\
& Puenting & 4x4 \\
& Paintball & Quads \\
& Barranquismo & Indoor Trainning \\
\hline $\begin{array}{l}\text { Actividades } \\
\text { acuáticas }\end{array}$ & $\begin{array}{l}\text { Piragüismo/kayak } \\
\text { Turismo fluvial }\end{array}$ & Rafting \\
& Buceo/submarinis- & Hidrobob/Hidros- \\
peed - Vela
\end{tabular}

Fuente: Vila et al. (2012)

En el Ecuador, el "Plan del Buen Vivir" menciona que la bicicleta puede ser utilizada para varias actividades humanas como el turismo. Por otro lado, Vila et al. (2012) lo complementa en su estudio "El turismo activo como modalidad turística en expansión define las categorías del turismo de naturaleza en subgrupos y las actividades que se practican dentro de cada campo", tal como se muestra en la tabla 1.

\section{Metodología}

El producto final se desarrolló a través de la recopilación y síntesis de tres productos elaborados. El primer producto trató los aspectos técnicos de las ciclo rutas de la Provincia del Guayas, en el segundo se levantó información sobre el perfil de ciclistas y sus necesidades, y para el tercero fue una 
revisión integral de los aplicativos de ciclismo más utilizados, que en conjunto con la información provista por los ciclistas, contribuyó al diseño de un aplicativo de cicloturismo. Adicionalmente, se muestran los resultados del nivel de aceptación, satisfacción, y recomendaciones por el uso del aplicativo diseñado.

\section{Aspectos técnicos de las ciclo rutas de la Provincia del Guayas}

En este primer informe se cuantificó las rutas de ciclismo y la frecuencia de recorridos de los ciclistas de la provincia del Guayas, mediante una investigación de campo plasmada en fichas técnicas para determinar sus características. Se consideraron 12 grupos pertenecientes a clubes de ciclismo formalizados, los cuales, en trabajo de campo durante un año, recopilaron la información de 51 rutas para ciclismo aplicado en la provincia del Guayas.

Se utilizó data de primer orden, la cual fue recopilada a través de entrevistas a turistas usuarios de las diferentes ciclo rutas de la provincia del Guayas. Se consideró una guía de preguntas para la entrevista a los líderes de los grupos de ciclismo. Además, se acompañó a los ciclistas a 36 rutas para observar las características de las mismas.

Como datos de segundo orden, se levantó información sobre turismo de naturaleza activo desarrollado en la provincia del Guayas, y se organizó conceptualmente a las diferentes ciclo rutas pertenecientes a la provincia del Guayas.

\section{Perfil de los tipos de ciclistas que recorren la provincia del Guayas}

El estado de la demanda de turismo activo de naturaleza se diagnosticó mediante una encuesta a una muestra de los ciclistas de la provincia del Guayas y la revisión documental de datos secundarios, para la elaboración de un perfil de los tipos de ciclistas que recorren la zona.

Se tomó como referente a la población de ciclistas de la provincia del Guayas, los cuales se estiman en 30,000 usuarios. El estudio se realizó a hombres y mujeres desde los 18 a los 65 años que usen la bicicleta en zonas urbanas y rurales. El tamaño muestral considerado fue de 384 individuos pertenecientes a 12 grupos, tomados durante 2 meses, en las distintas excursiones organizadas por los clubes de ciclismo.

En el presente estudio se busca determinar el perfil sociodemográfico, los hábitos deportivos y de tiempo libre; además de los intereses y motivaciones de los ciclistas que recorren la provincia del Guayas. En el aspecto técnico se utilizó una encuesta con 18 preguntas cerradas para la recopilación de la información de primer orden, la encuesta fue aplicada antes y después de los recorridos.

\section{Aspectos técnicos y de contenido que deben ser considerados en el diseño del aplicativo móvil de cicloturismo}

Se valoró los aspectos técnicos y de contenido que deben ser considerados para el diseño de un aplicativo móvil de cicloturismo, a partir de los resultados obtenidos en la investigación del perfil del ciclista de la provincia del Guayas, y de la comparativa con otras aplicaciones internacionales para determinar su factibilidad.

Se consideraron organizaciones de ciclismo registradas y reconocidas por la Dirección de Turismo de la Prefectura del Guayas y por otros clubes que participan en eventos de ciclismo auspiciados por tiendas que venden artículos para esta actividad. Se recopiló información a través de listas de apreciación con una escala valorativa para comparar los 4 aplicativos móviles de ciclismo más utilizados (strava, endomondo, runtastic, wikiloc) por 12 clubes de ciclismo que recorren la provincia del Guayas. En el aspecto técnico se utilizó una lista de apreciación con 3 categorías y 33 criterios para evaluar la funcionalidad, el contenido y la tecnología de los 4 aplicativos móviles de ciclismo que utilizan actualmente los ciclistas.

\section{Medición del nivel de satisfacción del usuario final del aplicativo piloto de cicloturismo}

La información fue recopilada a través de un cuestionario de 11 preguntas y con los resultados se aplicó el Cuadro Lógico de IADOV (Kuzmina, 1970; López y González, 2002) con el fin de evaluar la satisfacción de los usuarios con respecto al diseño del aplicativo de cicloturismo.

Como muestra se seleccionó un grupo compuesto por 17 ciclistas. La caracterización del grupo objeto de estudio fue la siguiente: hombres y mujeres entre 18 y 40 años, entre 1 y 16 años como ciclista, usan algún aplicativo móvil de ciclismo y además se consideró que todos realicen recorridos en bicicleta fuera de Guayaquil por lo menos $1 \mathrm{vez}$ al mes.

Se recolectó la data a través de un cuestionario de 11 preguntas y con los resultados se aplicó el Cuadro Lógico de IADOV. Además, se hicieron pruebas con el ingreso de información en el aplicativo móvil en la cual también participaron miembros de 7 clubes de ciclismo del Guayas.

El tipo de investigación es descriptiva con metodología cualitativa, lo que permitió conocer el grado de satisfacción de cada ciclista en relación al diseño del aplicativo móvil. Por ejemplo, si uno de los participantes contestó "No sé" en la pregunta 11 se ubica en la columna central de la tabla 3 y si contestó nuevamente "No sé" en la pregunta 10 entonces se busca en las filas del centro en las casilla donde aparece esa respuesta y se ubica el punto donde se intercepta con la fila "No me gusta tanto" con la columna "No sé". El resultado de dicho ciclista es " 3 ", que equivale a "satisfacción no definida". De esta forma se clasifican en seis categorías.

2. ¿Qué es lo que más le gusta del aplicativo? 
Tabla 2.Cuadro lógico de IADOV

11. ¿Utilizaría otro aplicativo móvil para informarse sobre cicloturismo en el Guayas?

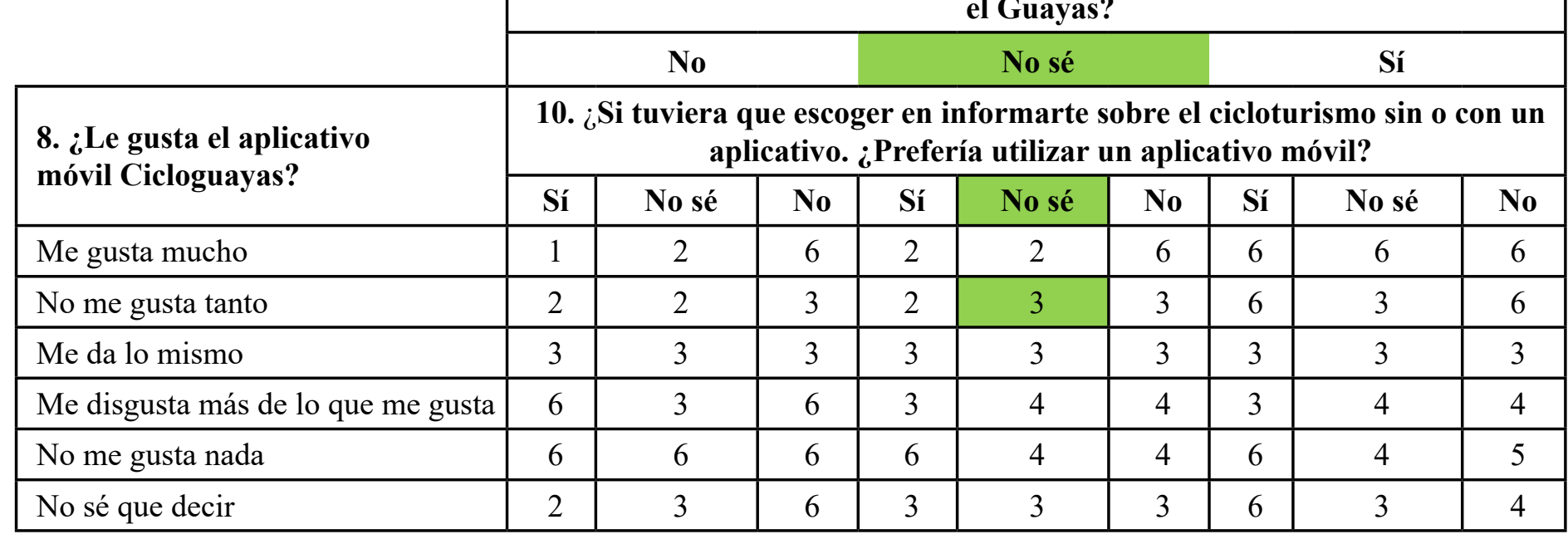

3. ¿Qué es lo que más le gusta del aplicativo?

Los criterios utilizados se fundamentan en las relaciones que se establecen entre tres preguntas cerradas que se intercalaron dentro del cuestionario y cuya relación los ciclistas desconocían. Además, también se utilizaron otras 2 preguntas abiertas que fueron complementarias, tal como se muestra en la tabla 2. El número resultante de la interrelación de las tres preguntas cerradas nos indica la posición de cada evaluador en los siguientes niveles de satisfacción:

1. Clara satisfacción

2. Más satisfecho que insatisfecho

3. No definida

4. Más insatisfecho que satisfecho

5. Clara insatisfacción

6. Contradictoria

De acuerdo a los resultados de cada cuestionario se aplicó la siguiente fórmula para calcular el índice de satisfacción grupal en referencia al diseño del aplicativo móvil de cicloturismo:

$I S G=\frac{A(+1)+B(+0,5)+C(0)+D(-0,5)+E(-1)}{N}$

El índice de satisfacción grupal es igual al número de ciclistas que evidenciaron una clara satisfacción (A) multiplicado por $(+1)$ más el número de ciclistas que se reflejaron más satisfechos que insatisfechos $(B)$ multiplicado por $(+0,5)$ más el número de ciclistas que se encontraban como no definidos o contradictorios (C) multiplicado por (0) más el número de ciclistas que indicaron que se encuentran más insatisfechos que satisfechos (D) multiplicado por $(-0,5)$ más el número de ciclistas que se demostraron una máxima insatisfacción (E) multiplicado por $(-1)$ y el resultado de todo se divide para el número total de la muestra.

- +1: Máxima Satisfacción (A).

- +0,5: Más satisfecho que insatisfecho (B).

- 0 : No definido y contradictorio (C).

$\cdot-0,5$ : Más insatisfecho que satisfecho (D).
- -1: Máxima insatisfacción (E).

El índice grupal de satisfacción arroja valores que van desde -1 hasta +1 . Los valores que se encuentran entre -1 y $-0,5$ denotan insatisfacción. Los comprendidos entre $-0,49$ y $+0,49$ indican contradicción. En cambio, los que resultan entre 0,5 y 1 evidencian satisfacción.

\section{Resultados}

A lo largo de este estudio se identificaron diferentes actores gubernamentales que promueven el turismo activo de naturaleza para la provincia del Guayas, uno de ellos es la Prefectura del Guayas. Esta institución llevó a cabo la primera guía de cicloturismo para aquellos turistas adeptos al ciclismo y turismo activo de naturaleza durante el año 2015. Esta guía fue elaborada con la ayuda de 12 grupos de ciclismo activos de la provincia del Guayas y presenta las diferentes alternativas de rutas (urbanas, rurales y mixtas), dificultad de las rutas propuestas (manejo, ascenso y distancia) y un listado de grupos de ciclismos activo que están presente en la provincia del Guayas.

La investigación realizada incluye también gustos, preferencias y necesidades de los ciclistas pertenecientes a la provincia del Guayas. En la misma se destaca el nivel educativo de los ciclistas, los cuales tiene un grado universitario en su mayoría y guarda relación con las investigaciones previas sobre ciclismo en otras regiones del mundo. Así también, coinciden otros factores como la práctica del ciclismo los fines de semana, salud, socialización, práctica de turismo (encuentro de flora y fauna, gastronomía, y comunitario). Además, se determinó que lo ciclistas de la muestra incurren en un gasto mensual promedio de $\$ 35$ y una gran mayoría posee celulares inteligentes y los usan durante sus recorridos.

El diseño de un aplicativo móvil fue propuesto para cubrir las necesidades y carencias de los aplicativos ya existentes. 
Además, se busca promover la comunicación entre los diversos grupos de ciclismo de la provincia del Guayas y otros en busca de turismo activo de naturaleza. El aplicativo móvil piloto fue elaborado para sistemas operativos Android que es el más común entre los ciclistas de la provincia. El mismo cuenta con 6 menús interactivos (noticias, eventos, galería, mapa, chat y número de emergencias). Además, tiene filtros para realizar búsquedas específicas de temas de interés entre los ciclistas como cicloturismo, competencias, tipos de rutas, eventos y agenda.

Finalmente, se realizó un estudio de satisfacción para verificar la aceptación del aplicativo piloto "CicloGuayas", el mismo que obtuvo un resultado destacado de satisfacción, de 0,53 por medio del método de IADOV.

\section{Conclusiones y Recomendaciones}

El diseño de un aplicativo móvil fue propuesto para cubrir las necesidades y carencias de los aplicativos ya existentes. Además, se buscó promover la comunicación entre los diversos grupos de ciclismo de la provincia del Guayas y otros en busca de turismo activo de naturaleza. El aplicativo móvil piloto fue elaborado para sistemas operativos Android que es el más común entre los ciclistas de la provincia. El mismo cuenta con 6 menús interactivos (noticias, eventos, galería, mapa, chat y número de emergencias). Además, tiene filtros para realizar búsquedas específicas de temas de interés entre los ciclistas como cicloturismo, competencias, tipos de rutas, eventos y agenda.

Después de realizar la presente investigación son pertinentes las siguientes recomendaciones:

- Integrar otras rutas a las 52 identificadas para expandir las opciones que actualmente tienen los ciclistas. Para próximas investigaciones se necesitaría un acercamiento hacia otros tipos de grupos no identificados que pertenecen a zonas alejadas de la provincia y que tengan inmersión con el desarrollo del turismo activo de naturaleza.

- Mejorar el diseño del aplicativo piloto en relación a la estética de la galería y el tiempo de carga del chat, tal como fue manifestado por algunos ciclistas que probaron su funcionalidad.

Para futuras líneas de investigaciones se podría identificar las características sociales y demográficas de las poblaciones aledañas a las ciclo rutas, para que los turistas puedan visitar los atractivos turísticos de la zona e incidir en la mejora de los ingresos económicos de la comunidad. Además, sería muy pertinente un estudio en el tiempo de la evolución de las zonas de influencia a las rutas que frecuentan los ciclistas del Guayas.

\section{Listado de Referencias}

Aguirre, M. (2014). Proyecto de implementación de ciclo rutas como atractivoturístico de la Parroquia el Valle. Recuperado de http://dspace.uazuay.edu.ec/bitstream/ datos/4018/1/10617.pdf

Brandenburg, C. (2007). Weather and cycling - a first approach to the effects of weather conditions on cycling. Recuperado de http://www.urbanclimate.net/matzarakis/ papers/Weatherandcycling.pdf

Consejería de Fomento y Vivienda de la Junta de Andalucía. (2014). Plan Andaluz de la bicicleta. Recuperado de http://www.juntadeandalucia.es/fomentoyvivienda/portalweb/web/texto/83770544-954a-11e2-8f48-a9c3a8bb53aa

Durán, A., Álvarez, J., y De la Cruz del Río, M. (2014). Active tourism research: a literature review (1975-2013). Recuperado de http://ruc.udc.es/dspace/bitstream/ handle/2183/14503/ROTUR_2014_08_art_5.pdf?sequence=1

Fas, E. (2015). Diseño de una ruta en bicicleta por la Sierra de Talayuelas (Cuenca, Castilla la Mancha, España) (Doctoral dissertation). Recuperado de http://riunet.upv. es/handle/10251/46861

Fernández-Latorre, F. (2015). Flujos turísticos, capital territorial y uso de la bicicleta. Andalucía como modelo de destino emergente en Cicloturismo. Revista de Estudios Andaluces No 32, pp. 76-107. DOI: $h t t p: / / d x$.doi.org/10.12795/rea.2015.i32.04

Foszto, M. (2014). The economic development from the perspective of cycle tourism in Covasna. Agricultural Management / Lucrari Stiintifice Seria I, Management Agricol, 16(4), 23-27.

Gilabert, J. (2015). Cicloturismo en la provincia de Alicante: análisis de la demanda y de los recursos sociales y territoriales. Recuperado de http://hdl.handle.net/10045/48114 Instituto Nacional de Estadísticas y Censos. (2014). Informe Demográfico.

Kormosne-Koch, K. (2013). Bicycle tourism in Hungary, Applied

Studies in Agribusiness and Commerce, Volume 07, Number 1, 2013. Recuperado de http://purl.umn.edu/152227.

Kuzmina, N.(1970). Metódicas investigativas de la actividad pedagógica. Editorial Leningrado.

Lee, C. (2014). An investigation of factors determining cycling experience and frequency. Tourism Geographies, 16(5), 844-862.

López, A. y González, V. (2002). La técnica de Iadov. Una aplicación para el estudio de la satisfacción de los alumnos por las clases de Educación Física. Lecturas Educación Física y Deportes, Revista Digital [en línea], 47. Buenos Aires.

Lumsdon, L. (2000). Transport and Tourism: Cycle Tourism - A Model for Sustainable Development. Recuperado de http://dx.doi.org/10.1080/09669580008667373

Meschik, M. (2012). Sustainable Cycle Tourism along the Danube Cycle Route in Austria. Tourism Planning \& Development, 9(1), 41-56.

Ministerio de Industrias, Turismo y Comercio de España. (2004). El turismo de naturaleza en España y su plan de impulso. Recuperado de http://www.altoalagon.es/ documentos/turismonaturaleza.pdf

Ministerio de Transporte y Obras Públicas. (2015). Plan Nacional de Ciclovías Recuperado de http://cicloviasecuador.gob.ec/quienes-somos.html.

Mintel. (2009). Cycling Holidays - UK. Disponible en

$U R L$

http://www.tandfonline.com/doi/abs/10.1080/09669580008667373

Prefectura del Guayas. (2012). Guía Turística de la Provincia del Guayas. Recuperado de http://www.guayas.gob.ec/turismo/guiasturisticas/es.html

Piket, P., Eijgelaar, E. y Peeters, P. (2013). European cycle tourism: a tool for sustainable regional rural development, Applied Studies in Agribusiness and Commerce $>$ Volume 07, Number 2-3, 2013. Recuperado de: http://purl.umn.edu/164816.

Pucher, J., Buehler, R., y Seinen, M. (2011): "Bicycling renaissance in North America? An update and re-appraisal of cycling trends and policies". Transportation Research Part A Policy and Practice, 45(6), 451-475. doi:10.1016/j.tra.2011.03.001.

Regional Tourism Organization 8. (2011). Cycle Tourism Assessment and Strategy Study.

Vila, N., Fraiz Brea, J. y Carril, V. (2012). El turismo activo como modalidad turística en expansión. Análisis de la oferta de turismo activo en España. (Spanish). Contabilidad Y Negocios, 7(13), 59-70.

Vujko, A., Plavša, J. y Ostojić, N. (2014). Impact of the "Danube cycling route" on the development of cycling tourism in Serbia, Polish Journal of Sport and Tourism. Volume 20, Issue 3, Pages 227-233, ISSN (Online) 2082-8799, ISSN (Print) 1899-1998, DOI: 10.2478/pjst-2013-002

Wadud, Z. (2014). Cycling in a changed climate. Journal of Transport Geography, 35, 12-20.

Wang, G., Macera, C. A., Scudder-Soucie, B., Schmid, T., Pratt, M., \& Buchner, D. (2005). A cost-benefit analysis of physicalactivity using bike/pedestrian trails. Health promotion practice, 6(2), 174-179.

Weston, R., Davies, N., Peeters, P., Eijgelaar, E., Lumsdon, L., McGrath, P., \& Piket, P. (2012). The European cycle route network Eurovelo: Challenges and opportunities for sustainable tourism. Update of the 2009 study. Brussels, Belgium: European Parliament, Directorate General for Internal Policies, Policy Department B: Structural and Cohesion Policies, Transport and Tourism.

Zovko, I. (2013). The Value of Cycle Tourism. Transform Scotland.

Disponible en: http://www.transformscotland.org.uk/wp/wp-content/uploads/2014/12/

The-Value-of-Cycle-Tourism-full-report.pdf 